\title{
Current status of the torus palatinus and torus mandibularis
}

\author{
Andrés S. García-García ${ }^{1}$, José-María Martínez-González ${ }^{2}$, Rafael Gómez-Font ${ }^{3}$, Ángeles Soto-Rivadeneira ${ }^{4}$, \\ Lucia Oviedo-Roldán 5
}

${ }^{1}$ Master in Oral Surgery, Implantology and Periodontics from the University of Southern Mississippi

${ }^{2}$ Full Professor of Oral Surgery. Complutense University of Madrid

${ }^{3}$ Director of the Master of Oral Surgery at the European University of Madrid

${ }^{4}$ Master in Oral Surgery, Implantology and Periodontics from the University of Southern Mississippi

${ }^{5}$ Master in Oral Surgery, Implantology and Periodontics from the University of Southern Mississippi

Correspondence:

University of Madrid. Spain

jmargo@odon.ucm.es

Received: $05 / 05 / 2008$

Accepted: $31 / 07 / 2009$

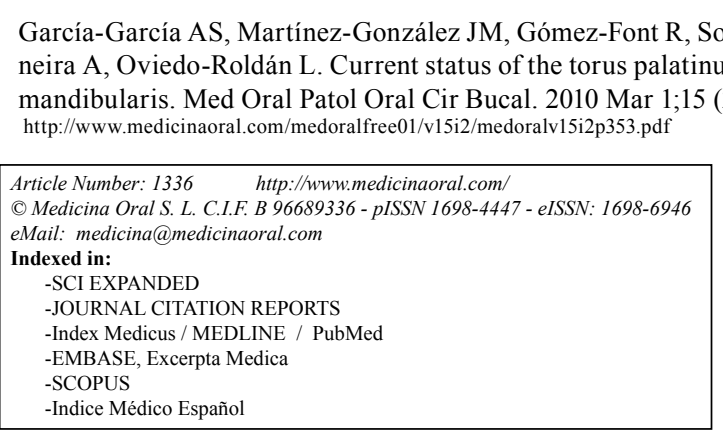

\begin{abstract}
While there is a hereditary component to tori, this does not explain all cases. Tori tend to appear more frequently during middle age of life; the torus palatinus is more commonly observed in females, but this is not the case with the torus mandibularis. Certain ethnic groups are more prone to one torus or the other. The torus is mainly removed due to prostodontic reasons, as it may also be used as biomaterial, not only in periodontology, but also in implantology. The aim of this study was a review of the literature from the past twenty years.
\end{abstract}

Key words: Torus palatinus, torus mandibularis.

\section{Introduction}

The tori (meaning "to stand out" or "lump" in Latin) (1) are exostosis that are formed by a dense cortical and limited amount of bone marrow, and they are covered with a thin and poorly vascularized mucosa. They are usually located at the longitudinal ridge of the half palatine, on the union of the palatine apophysis of the maxillae or on the internal side of the horizontal branch of the jaw, above the mylohyoid line and at the level of the premolar area (1-8) and canine area $(6,8-10)$, presenting a very slow $(1,2)$ and progressive growth that can stop spontaneously (1).

Castro Reino et al. (1) define it as a congenital bony protuberance with benign characteristics, leading to the "overworking" of osteoblasts and bone to be deposited along the line of fusion of the palate or on the hemimandibular bodies.
The discovery of these exostoses usually occurs incidentally during a routine clinical exam, as they usually do not produce any symptoms, except in cases of significant growth or in edentulous patients, in which case they may hinder the construction of the prosthesis. Despite the numerous studies of which they have been and continues to be the focus, their origin is unclear; various possible causes are presented in the literature, but none of them are definitive. A certain prevalence with respect to ethnic groups, sex and age has also been observed. Its elimination in dentate patients cannot be justified, unless it can be used clinically or as a filling biomaterial in order to correct bone defects that patients may suffer in some part of their jaw area.

The prevalence of occurrence of the tori, according to the study by Al-Bayaty et al. (4) is $12.3 \%$, very close to 
that of Bruce et al. (7) with $14.6 \%$, while Jainkittivong et al. (6) showed their prevalence to be $26.9 \%$.

Since there is a very limited amount of current scientific literature that discusses the tori, given that they are very common and have no pathological interest, we aim to conduct a review of the literature of the past 20 years, in order to analyze the etiological and epidemiological factors, as well as its treatment, complications and possible application in the field of bone regeneration.

\section{Etiology}

The exact cause of appearance of the tori is not clear. $(4-6,9,11)$ The most widely accepted theory today is genetics $(1,2,4-7,11,12)$, but it has not always been possible to show the autosomal dominant nature of its appearance. $(7,14)$ In the three clinical cases analyzed by Curran et al. (15), in which a daughter, mother and grandmother had autosomal dominant osteosclerosis, mandibular tori (MT) and palatine tori (PT) were found to be present in all three women. In the study by Eggen (16), it was only possible to estimate the genetic origin of the TM in $29.5 \%$ of the cases; as for the rest of the cases, approximately $70 \%$, the origin was attributed to environmental factors, mainly related to occlusal stress.

Another cause is superficial injuries $(1,2)$ or its occurrence as a functional response in individuals with welldeveloped chewing muscles, $(2,4-6,11,13,16)$ or in patients with abraded teeth due to occlusion. (2, 5-7, 11-13, 16,17) In the study conducted by Reichart et al. (3), they found a significant correlation between the incidence of torus and the presence of abraded teeth in Thais, but not in Germans. In studies conducted by Sirirungrojying et al. (5), Clifford et al. (10) and Kerdpon et al. (12), they found a large relationship between the TM and parafunctional habits, which they did not find with the TP. $(5,12)$ Thus, Sirirungrojying et al. (5) determined that TM can be used to indicate the risk of appearance of temporomandibular disorders.

As possible causes, other authors mention eating habits, $(4,5,7)$ states of vitamin deficiency or supplements rich in calcium (13), and also diet (4, 16-19). In the studies conducted by Eggen et al. (18) and Al-Bayaty et al. (4), they associate the consumption of fish with the presence of tori, because fish contains $\Omega 3$ unsaturated fatty acids and vitamin $\mathrm{D}$, encourages bone growth.

Sasaki et al. (20) tried to draw a relationship between the occurrence of tori and the prolonged use of phenytoin, but were unable to conclude that it was what caused the appearance of the tori. However, they did determine that it is a factor that leads to an increase in size, because it induces an increase in calcium homeostasis, functioning as an osteogenic agent.

Lastly, and possibly maintaining a certain relationship with the aforementioned injuries, Sonnier et al. (11) as- sociate the presence of teeth at the mandibular level with the presence torus. To further support this, in the study conducted by Eggen et al. (21), they found a relationship between the presence of torus and the number of teeth present in the mouth. In addition, among adolescents with mandibular tori, the ratio of non-erupted canines was much smaller than those who did not have any tori. Furthermore, in another study, Eggen (19) found a relationship between the presence of mandibular tori and a normal bone height around the teeth.

\section{Frequency}

As aforementioned, the prevalence of the appearance of tori is very important, which according to the study of Al-Bayaty et al. (4), is $12.3 \%$, very close to the $14.6 \%$ found by Bruce et al. (7).

In the majority of the studies reviewed, the appearance of the TP is more frequent than that of the TM, (3-6, $12,14,18,22-25)$; however, there are reviews such as those by Sonnier et al. (11), Sirirungrojying et al. (5) and Bruce et al. (7), which show a higher presence of TM than of TP.

The only studies that we found linking the presence of both exostosis were by Al-Bayati et al. (4), Bruce et al. (7) and Haugen (22), who reported it in $2 \%$ to $3 \%$ of cases, whereas Jainkittivong et al. (6) reported its presence in $28.12 \%$ of the cases. Haugen (22) found that the probability of finding TM in a person with TP is more than double than that of a person without TP, and vice versa. In their study of Trinidad and Tobago, Al-Bayaty et al. (4) also believe that there is a strong association between TP and TM (50\% of subjects with TM had TP, yet only $30 \%$ of subjects with TP had TM) (Table 1).

\section{Age}

It is not easy to compare the range of ages provided in the studies that we have analyzed, since in many cases, they are not standardized and each author gives a different reference. According to a study by Bruce et al. (7), the average age when experiencing the onset of tori, is 34 years old. According to Al-Bayaty et al. (4), the average age is 30.7 years old for patients with TP and 39.2 years old for those with TM.

The onset of tories appears to be earlier among those with TP $(4,13)$, where cases of such onset have been described from birth and the first decade of life. (13) In the observations of Reichart et al. (3) (in women) and Al-Bayaty et al. (4) the most common age range for the onset of TP is from 11 to 20 years old. Although most studies found the more common age range for such onset to be between 30 and 50 years old, third and fourth decade of life $(3,7,12,14,22)$, in the study conducted by Haugen et al. (22), the age range that experienced the most frequent onset reached as high as 65 years old. According to Edmund et al. (26), TP appears during puber- 


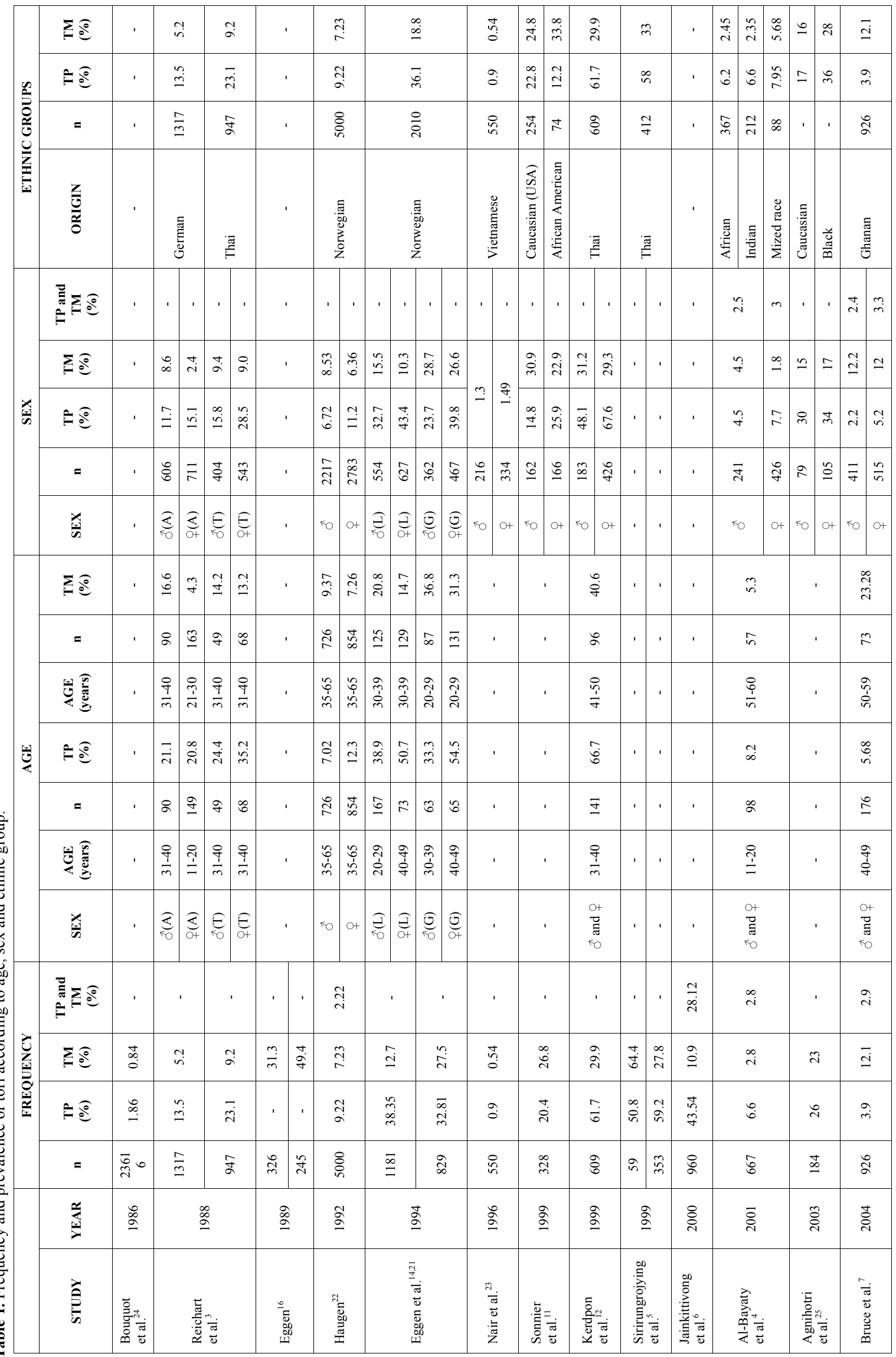


ty and slowly grows until the subject reaches adulthood, with the possibility of their growth continuing until the seventh decade of life.

The appearance of TM is rare before the first decade of life $(4,6,7)$. Apart from that, there is not much variation with respect to age of onset of TP; the only exception worth noting is that of the work of Al-Bayaty et al. (4), Bruce et al. (7) and Haugen (22), who extend the age range until the sixth decade of life. For Sonnier et al. (11), the prevalence of TM is inversely proportional to age (Table 1).

\section{Sex}

It is most frequent for TP to appear in women than in men $(1,2,8,13,18)$, and it is believed that there may be a dominant type linked to the $X$ chromosome. (1) In all the studies reviewed, there is a higher presence of TP in females than in males, although not all the studies noted this difference to be significant. $(3,4,7,11,12,14,22,23)$.

As for the TM, some authors have found no significant differences between men and women in their studies (13), although in all of the studies, it is more common in males.

The study conducted by Nair et al. (23) refers to the presence of torus in general, without differentiating between TP and TM, and does not find any significant differences between the two.

Only two studies offer results on the joint appearance of TP and TM in men and women, and both results are similar, although the difference is not significant $(4,7)$. (Table 1).

\section{Ethnic Groups}

The appearance of tori is more common in certain ethnic groups and countries (Eskimos, Japanese and in the United States) (2). For example, a great predisposition towards the appearance of TM has been observed among Mongols (13).

We have reviewed several articles with studies on different regions of the world. In the study by Sonnier et al. (11) in which they compare the incidence of torus among North Americans, and African Americans $(33.8 \%)$ were among those who presented the highest incidence of TM, whereas Caucasians (22.8\%) were more likely to present TP. In analyzing the incidence among Norwegians, we found two studies that give us different results. In the first study, the percentage of TP was $9.22 \%$ (22), whereas in the other study, TP was observed to be $36.1 \%$ (14) and TM was observed to be $7.23 \%$ and $18.8 \%$ respectively. The same is true in two other studies in Thailand. In the first study, the percentage of TP was $23.1 \%$ (3), and in the second study, it was $58 \%$ (5), whereas the incidence of TM was $9.2 \%$ and $33 \%$ respectively.

The populations in which we found elevated rates of occurrence of tori were among North Americans (Caucasians and African Americans) (11\%), Norwegians $(14.22 \%)$ and Thais (3.5\%) (Table 1).

\section{Size}

The growth of the tori is gradual, being greater in the second or third decade of life. $(7,20)$ As for this section, among the studies reviewed, there is no consensus on how to classify the growth; each study classifies the growth differently.

Haugen (22) and Eggen et al. $(14,16,18)$ classify the growth in terms of small, medium and large, less than 2 $\mathrm{mm}, 2$ to $4 \mathrm{~mm}$ and more than $4 \mathrm{~mm}$, respectively. $(16,18)$ Thus, according to the study conducted by Haugen (22), the majority of the TP were small, with $69.85 \%$ (322) of the TP found in $6.44 \%$ of individuals. The growth was also small for the TM, and was found in $60.11 \%$ (220) of the TM, or $4.40 \%$ of the individuals. Many times, because these exostoses are so small, they go unnoticed in the mucous coating. In the studies conducted by Eggen, the small TM were also the most numerous, both in the study group, 59.5\% (72), as well as in the control group, $66.7 \%$ (68) (16); as for the TP, the small TP were the most frequent, 91\% (659) (18).

Another classification is that performed by Reichart et al. (3), which classifies them as grade 1, small up to 3 mm; grade 2, moderate up to $6 \mathrm{~mm}$; and grade 3 , marked above $6 \mathrm{~mm}$. The size most often found in the TP of the Germans and Thai was observed to be small in $84.9 \%$ and $94.9 \%$ of the cases, respectively. Interestingly, German men were only observed to have TP of this size. As for the TM, the size that was most common according to their study was small, with $88.4 \%$ and $82.8 \%$ in Germans and Thais, respectively.

The studies conducted by Sonnier et al. (11) and Al-Bayaty et al. (4) coincide very little on the average measurements of the studies they conducted on TP. The average measurements were 20.33 x $9.45 \mathrm{~mm}$ and $21 \times 18 \mathrm{~mm}$ respectively, and they match up significantly more for the TM, $10.9 \times 6.49 \mathrm{~mm}$ and $10 \times 9 \mathrm{~mm}$ respectively. The study conducted by Sirirungrojying et al. (5) attempted to link the size of the tori with the incidence of parafunction, but were unable to find a relationship.

\section{Shapes}

There is a lot of variety in terms of shapes; the TP can be flat, nodular, lobular or spindle-shaped $(3,4,8,18,22)$, and the TM are usually nodular, unilateral or bilateral and single or multiple. $(3.4,9-11,22)$ (Fig. 1).

As it regards the shape of the TP, there is no overlap of results in the articles reviewed. In the study conducted by Haugen (22), the most common shape was small and nodular; in most cases, the more voluminous TP were nodular, whereas the lobular shapes were more rare. In the study by Reichart et al. (3), the most frequent shape 


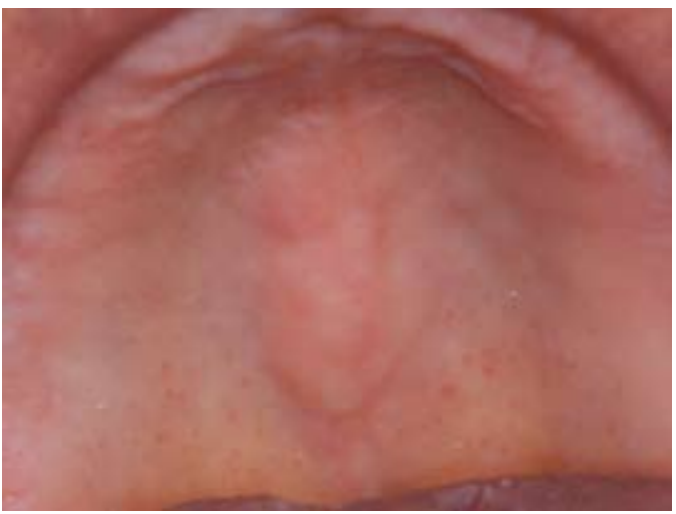

Fig.1. Palatine tori.

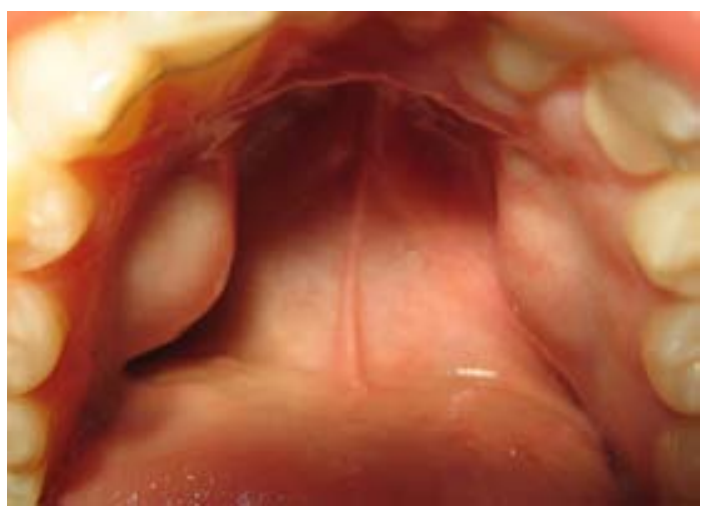

Fig. 2. Mandibular tori.

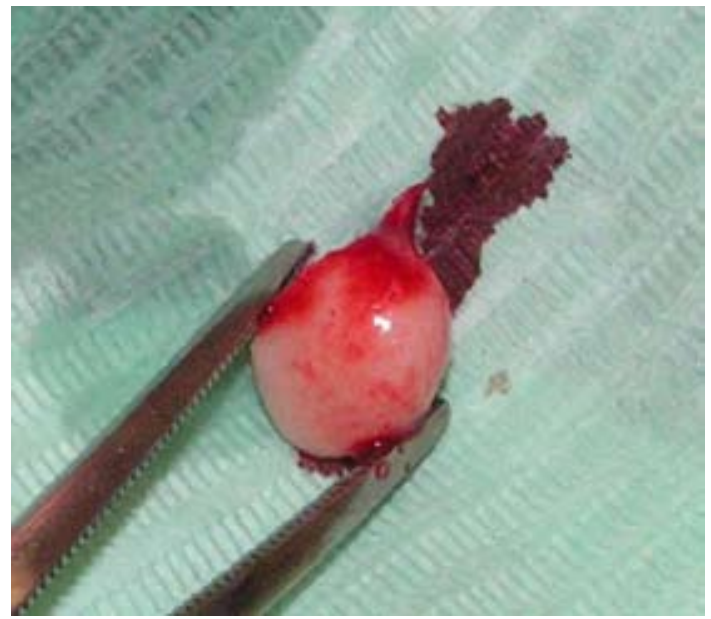

Fig. 3. Mandibular tori as grafts in order to place implants.

among the Saxon population was that of the spindleshape (39.8\%), with the nodular shape not trailing very far behind (37.6\%). In the study by Al-Bayaty et al. (4), in the majority of cases $48 \%$ (30), the most common shape was flat.

As for the TM, it was found that the majority of studies agree that the most frequent shape in which they appear is bilateral $(3,4,7,9,11,22)$. In the study by Al-Bayaty et al. (4), the most common shape was nodular in $61 \%$ (23) of the cases, and bilateral in $87 \%$ (33) of the cases of TM. This shape was also the most common in both German and Thai (52.2\% and $87.35 \%$ respectively), in the study by Reichart et al. (3), as well as in the studies conducted by Sonnier et al. (11) (73.9\%) (Fig. 2).

\section{Diagnosis}

In most cases, the finding is usually incidental and observed during clinical examination at the dental office. This is because they are asymptomatic for the most part, and those who have torus are not aware of it $(1,4)$. Sometimes patients may present phonatory disturbances, limitation of masticatory mechanics, ulcerations of the mucosa, food deposits, prosthetic instability, and some patients may experience cancerophobia, and consult a professional in order to look for a solution (13).

They are diagnosed by clinical examination:

The torus palatinus can be unilobular, polylobulated, flat and spindle-shaped, located at the midline of the hard palate $(2,13)$.

The torus mandibularis are usually symmetrical and bilateral $(1,2)$, but can also be unilateral (1), located on the lingual side of the mandible, above the mylohyoid line and at the level of the premolars $(4,13)$.

$\mathrm{X}$-rays taken reveal radiodense images with a slightly higher density than that of the surrounding bone. $(1,13)$ Carrying out X-rays (periapical, occlusal, and panoramic) is not very useful, given the simplicity of the diagnosis during clinical examination (13).

Histopathological examination reveals that it is similar to the compact structure of the normal bone, having a slightly spongy structure with marrow spaces (1).

\section{Treatment}

Removal of the tori is not always necessary. The most frequent cause of extirpation continues to be the need for prosthetic treatment $(1,2,11)$ or that of being a potential source of autogenous cortical bone for grafts in periodontal surgery, cyst surgery or implant surgery $(9,11,27,28)$ (Fig.3), although long-term stability of the grafts is uncertain (Table 2).

Barker et al. (9) used the bone obtained from the TM of a patient in order to increase the thickness of his upper jaw and allow the placement of implants, in order to replace the lateral incisors and absent canines. They determined that the TM provided a useful and local source of bone in procedures for increasing bone thickness. Proussaefs (28) discusses three clinical cases in which he makes a clinical and histological evaluation of the use of mandibular tori as grafts in order to place implants, in which he obtained up to a $4.33 \mathrm{~mm}$ increase in thickness and an absorption rate similar to the grafts 
that are made with bone from other donor areas of the oral cavity.

Other authors do not recommend the removal of tori except in very extreme cases, and they recommended the removal of the prosthesis in these areas (2) or the use of soft acrylics on the edges of the prosthesis (29). We can now avoid their removal while offering patients another alternative for rehabilitating the jaw by means of a prosthetic implant.

\section{Surgical technique and complications}

The instruments used will be that typically used in oral surgery (with a chisel and / or burrs), with local or general anesthesia, depending on the case, although local anesthesia is sufficient in most cases (1).

For the removal of the TP, the nasopalatine nerve must be anesthetized at its exit through the anterior palatine foramen, and the anterior palatine nerves must be anesthetized through the posterior palatine foramen. In addition, the anesthesia will be administered by perilesional infiltration in order to facilitate the detachment of the palatine fibromucosa (1).

To eliminate the TM, Castro Reino et al. (1) recommend administering the anesthetic by means of infiltration in the lesion; however, in our service, we recommend a nerve block anesthesia of the inferior alveolar nerve, and also the lingual and mental nerves.

The use of general anesthesia is not indicated for this type of surgery, given that it poses a risk to the patient, and based on the fact that its systematic use is not justified (1).

Different incisions can be made in order to perform the removal of the TP. The most common type of incision used is the double-Y incision, because it prevents injury of the nasopalatine and anterior palatine blocks $(1,2)$. The incision will be a total thickness (1).

To treat the TM, an incision will be made on the mandibular ridge, with an incision made above the torus, which provides us with a good operating field (2), or scalloped following the necks of the teeth (when they are present) along the tongue, sectioning the gingival ligament $(1,2)$.

Periostotomes will be used for the detachment, and we will separate the fibromucosa until the lesion is exposed (1).

As aforementioned, the mucosa that covers the torus is very thin, which makes it tear easily if we are not careful (1).

The flaps can be anchored by suturing to the teeth, or by separating them using Farabeuf in order to avoid damaging them while performing the operation (1).

In the TM, a piece of gauze may be placed between the lingual flap and the surgical space that extends under the torus. This will prevent the dried bone from becoming lost deep within the structures of the mouth (1).
Initially, the excision will be performed using a fissure bur, which we use to divide the torus; then we will use a chisel to cut into each of the divisions, or alternatively, a large burr or a file may be used to reshape the bone or to even it out. $(2,26)$ If the torus is not very large, it is not necessary to divide it; it can be removed directly with a burr. Castro Reino et al. (1) advocate the use of a high speed turbine cooled with normal saline solution, given that they consider the use of a chisel and hammer involves a greater risk of iatrogenic injury, and also to avoid the bumping the patient with the chisel; however, we must take into account the risk that this may cause emphysema.

By removing the torus and relocating the flaps, we will end up with too much soft tissue, which can be removed with scissors. The suture may be made by simple points, mattress sutures, etc., provided they are not too tight (1).

Surgical cement is used to protect the wound from traumatic and mechanical force during the healing process, so as to achieve a bacteriostatic or bactericidal action if it contains an agent with these properties, and to prevent the accumulation of food remains (1).

In the TP, a surgical splint should be placed over the surgical cement for a period of 48 hours, and is removed when reviewing the wound. The patient's former relined and adjusted prosthesis can also be reseated $(1,2)$.

Although it is uncommon, complications can occur as a result of iatrogenic maneuvers on the part of the professional $(1,2)$ (Table 2).

\section{Post-operative care}

The patient must be informed that the signs and symptoms that may occur during the postoperative period will be those that are most commonly associated with this type of surgical procedure, such as edema, hematoma, mild pain, etc. Postoperative medication will consist of antibiotics, analgesics and anti-inflammatory medicine, as well as stressing that it is important for the patient to continue with appropriate personal hygiene so that the wound may heal properly (1). 
Table 2. Causes of exeresis and complications of the tori.

\begin{tabular}{|c|c|c|c|}
\hline $\begin{array}{l}\text { CAUSES FOR CARRYING OUT } \\
\text { EXERESIS OF THE TORUS }\end{array}$ & & $\begin{array}{c}\text { SURGICAL COMPLICATIONS } \\
\text { OF THE TORI }\end{array}$ & $\begin{array}{l}\text { POST-OPERATIVE } \\
\text { COMPLICATIONS } \\
\text { OF THE TORI }\end{array}$ \\
\hline \multirow{2}{*}{$\begin{array}{l}\text { - Disturbances of phonation }{ }^{1,13,22,26} \\
\text { - Limitation of masticatory mechan- } \\
\text { ics }^{1,13,22,26} \\
\text { - Sensitivity due to the thin mucosa } \\
\text { layer }{ }^{1} \\
\text { - Traumatic inflammation }{ }^{1} \\
\text { - Ulcer of a traumatic origin }{ }^{1,2,13,26} \\
\text { - Retention of food remains }{ }^{1,2,13} \\
\text { - Esthetic reasons }{ }^{1} \\
\text { - Prosthetic instability }{ }^{2,13,22} \\
\text { - Patients with cancerophobia } \\
\text { - Prosthetic treatment }{ }^{1,2,4,11,22,26} \\
\text { - Source of autogenous cortical bone } \\
\text { for grafts }{ }^{11}\end{array}$} & 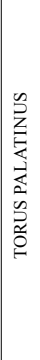 & $\begin{array}{l}\text { - Perforation of the nasal cavities }{ }^{1,2} \\
\text { - Secondary anesthesia due to palatine } \\
\text { nerve damage }{ }^{1} \\
\text { - Bone necrosis due to poor refrigeration } \\
\text { during surgical drilling }{ }^{1} \\
\text { - Hemorrhage due to section of palatine } \\
\text { arteries } \\
\text { - Dilaceration of the palatine mucosa } \\
\text { - Fracture of the palatine bone }\end{array}$ & \multirow[t]{2}{*}{$\begin{array}{l}\text { - Hematoma }^{1,2,9,13} \\
\text { - Edema }{ }^{1,2} \\
\text { - Opening of a suture }{ }^{1,13} \\
\text { - Infection }{ }^{1,2,9,13} \\
\text { - Bone and mucosal necrosis }{ }^{1,13} \\
\text { - Neuralgia }{ }^{1} \\
\text { - Poor scarring }^{1,2}\end{array}$} \\
\hline & & 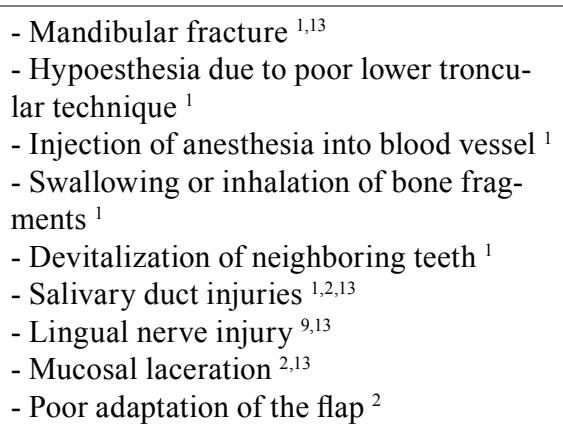 & \\
\hline
\end{tabular}

\section{References}

1. Castro Reino O, Perez Galera J, Perez Cosio Martin J, Urbon Caballero J. Surgical techniques for the exeresis of torus, both palatal and mandibular. We observe indications, contraindications and complications as well as enumerate all the right events to realize correctly the said techniques. [Surgery of palatal and mandibular torus]. Rev Actual Odontoestomatol Esp. 1990;50:47-50, 53-6.

2. Donado M. Pre-prosthetic Surgery. In: Donado M, ed. Cirugía bucal. Patología y técnica. [Oral Surgery. Pathology and Technique] 2th ed. Barcelona: Masson; 1998. p. 481-510.

3. Reichart PA, Neuhaus F, Sookasem M. Prevalence of torus palatinus and torus mandibularis in Germans and Thai. Community Dent Oral Epidemiol. 1988;16:61-4.

4. Al-Bayaty HF, Murti PR, Matthews R, Gupta PC. An epidemiological study of tori among 667 dental outpatients in Trinidad \& Tobago, West Indies. Int Dent J. 2001;51:300-4.

5. Sirirungrojying S, Kerdpon D. Relationship between oral tori and temporomandibular disorders. Int Dent J. 1999;49:101-4.

6. Jainkittivong A, Langlais RP. Buccal and palatal exostoses: prevalence and concurrence with tori. Oral Surg Oral Med Oral Pathol Oral Radiol Endod. 2000;90:48-53.

7. Bruce I, Ndanu TA, Addo ME. Epidemiological aspects of oral tori in a Ghanaian community. Int Dent J. 2004;54:78-82.

8. Antoniades DZ, Belazi M, Papanayiotou P. Concurrence of torus palatinus with palatal and buccal exostoses: case report and review of the literature. Oral Surg Oral Med Oral Pathol Oral Radiol Endod. 1998;85:552-7.

9. Barker D, Walls AW, Meechan JG. Ridge augmentation using mandibular tori. Br Dent J. 2001;190:474-6.

10. Clifford T, Lamey PJ, Fartash L. Mandibular tori, migraine and temporomandibular disorders. Br Dent J. 1996;180:382-4.

11. Sonnier KE, Horning GM, Cohen ME. Palatal tubercles, palatal tori, and mandibular tori: prevalence and anatomical features in a U.S. population. J Periodontol. 1999;70:329-36.

12. Kerdpon D, Sirirungrojying S. A clinical study of oral tori in southern Thailand: prevalence and the relation to parafunctional activity. Eur J Oral Sci. 1999;107:9-13.
13. Martínez-González JM. Tumores benignos de los maxilares [Benign tumors of the maxilla]. In: Donado M, ed. Cirugía Bucal. Patología y Técnica. 2th ed. Barcelona: Masson; 1998. p. 627-639.

14. Eggen S, Natvig B. Concurrence of torus mandibularis and torus palatinus. Scand J Dent Res. 1994;102:60-3.

15. Curran AE, Pfeffle RC, Miller E. Autosomal dominant osteosclerosis: report of a kindred. Oral Surg Oral Med Oral Pathol Oral Radiol Endod. 1999;87:600-4.

16. Eggen S. Torus mandibularis: an estimation of the degree of genetic determination. Acta Odontol Scand. 1989;47:409-15.

17. Eggen S, Natvig B. Variation in torus mandibularis prevalence in Norway. A statistical analysis using logistic regression. Community Dent Oral Epidemiol. 1991;19:32-5.

18. Eggen S, Natvig B, Gåsemyr J. Variation in torus palatinus prevalence in Norway. Scand J Dent Res. 1994;102:54-9.

19. Eggen S. Correlated characteristics of the jaws: association between torus mandibularis and marginal alveolar bone height. Acta Odontol Scand. 1992;50:1-6.

20. Sasaki H, Ikedo D, Kataoka M, Kido J, Kitamura S, Nagata T. Pronounced palatal and mandibular tori observed in a patient with chronic phenytoin therapy: a case report. J Periodontol. 1999;70:445-8.

21. Eggen S, Natvig B. Relationship between torus mandibularis and number of present teeth. Scand J Dent Res. 1986;94:233-40.

22. Haugen LK. Palatine and mandibular tori. A morphologic study in the current Norwegian population. Acta Odontol Scand. 1992;50:65-77.

23. Nair RG, Samaranayake LP, Philipsen HP, Graham RG, Itthagarun A. Prevalence of oral lesions in a selected Vietnamese population. Int Dent J. 1996;46:48-51.

24. Bouquot JE, Gundlach KK. Oral exophytic lesions in 23,616 white Americans over 35 years of age. Oral Surg Oral Med Oral Pathol. 1986;62:284-91.

25. Agnihotri N, Agnihotri J. Prevalence of oral tori in subjects requiring prothodontic care. New Jersey Dental School. University of Medicine \& Dentistry of New Jersey. 2003. Available at: http://www. dentalschool.umdnj.edu.

26. MacInnis EL, Hardie J, Baig M, Al-Sanea RA. Gigantiform To- 
rus palatinus: review of the literature and report of a case. Int Dent J. 1998;48:40-3.

27. Ganz SD. Mandibular tori as a source for onlay bone graft augmentation: a surgical procedure. Pract Periodontics Aesthet Dent. 1997;9:973-82.

28. Proussaefs P. Clinical and histologic evaluation of the use of mandibular tori as donor site for mandibular block autografts: report of three cases. Int J Periodontics Restorative Dent. 2006;26:43-51.

29. Abrams S. Complete denture covering mandibular tori using three base materials: a case report. J Can Dent Assoc. 2000;66:494-6. 